\title{
Characteristics of a Subset of Achalasia With Normal Integrated Relaxation Pressure
}

\author{
Eunju Kim, ${ }^{1}$ In Kyung Yoo, ${ }^{1}$ Dong Keon Yon, ${ }^{2}$ Joo Young Cho, ${ }^{1}$ and Sung Pyo Hong ${ }^{1 *}$ \\ Departments of ${ }^{1}$ Gastroenterology and ${ }^{2}$ Pediatrics, Cha Bundang Medical Center, Cha University College of Medicine, Seongnam, Gyeonggi-do, \\ Korea
}

\section{Background/Aims}

Integrated relaxation pressure (IRP) is a critical diagnostic criterion to define achalasia. However, there are some cases with typical symptoms and signs of achalasia but with normal IRP. The aim of this study is to evaluate the clinical characteristics of patients with achalasia with normal IRP and outcomes after peroral endoscopic myotomy (POEM).

\section{Methods}

Patients with achalasia were collected in whom POEM was performed from November 2014 to April 2018 at CHA Bundang Medical Center. Achalasia with normal IRP was defined by findings compatible to achalasia in Eckardt score, endoscopy with endoscopic ultrasound, high-resolution manometry, impedance planimetry (EndoFlip), and timed esophagogram.

\section{Results}

POEM was performed in 89 patients with achalasia; among them, 24 (27\%) patients were diagnosed with achalasia with normal IRP. Patients with achalasia with normal IRP were older, had longer duration of symptom, and had a more tortuous esophagus. In EndoFlip, the distensibility index and cross-sectional area were higher in patients with normal IRP. Therapeutic outcomes showed no statistically significant differences. On correlation analysis, IRP had negative correlations with age, disease duration, and distensibility index.

\section{Conclusions}

Patients with achalasia of normal IRP value were older and had longer disease duration and higher distensibility index and crosssectional area than patients with achalasia with abnormal relaxation of lower esophageal sphincter. Therapeutic outcomes were not different between the 2 groups.

\section{(J Neurogastroenterol Motil 2020;26:274-280)}

\section{Key Words}

Esophageal achalasia; Esophageal sphincter, lower; Myotomy

Received: June 24, 2019 Revised: August 25, 2019 Accepted: September 20, 2019

(a) This is an Open Access article distributed under the terms of the Creative Commons Attribution Non-Commercial License (http://creativecommons. org/licenses/by-nc/4.0) which permits unrestricted non-commercial use, distribution, and reproduction in any medium, provided the original work is properly cited.

*Correspondence: Sung Pyo Hong, MD

Division of Gastroenterology, Department of Medicine, CHA Bundang Medical Center, CHA University School of Medicine, 59 Yatap-ro, Bundang-gu, Seongnam, Gyeonggi-do 13496, Korea

Tel: +82-31-780-5641, Fax: +82-32-780-5005, E-mail: sphong@cha.ac.kr

Eunju Kim and In Kyung Yoo contributed equally to this work. 


\section{Introduction}

Achalasia is a rare esophageal motility disorder with an incidence of 0.5 cases per 100000 persons per year and a prevalence of 10 cases per 100000 persons. ${ }^{1,2}$ It is characterized by impaired lower esophageal sphincter (LES) relaxation and loss of peristalsis in the esophageal body. ${ }^{3}$ Patients with achalasia present with dysphagia, chest pain, regurgitation, and weight loss. ${ }^{4}$ The pathophysiology of achalasia has not been fully elucidated. A generally accepted mechanism is the functional or pathologic loss of myenteric plexus ganglion cells at the LES, leading to imbalance between the inhibitory and excitatory nerve distributions of the LES. ${ }^{5,6}$

For the diagnosis of achalasia, many modalities are used, such as upper endoscopy and timed barium esophagography. According to the Chicago classification, for the diagnosis of achalasia and other esophageal motility disorders, the gold standard tool is high-resolution manometry (HRM). Typically, incomplete LES relaxation and absence of esophageal peristalsis are noted at HRM. Integrated relaxation pressure (IRP) is an important diagnostic criterion to define achalasia.

However, there are some cases with typical signs and symptoms of achalasia but with normal IRP. Ponds et al ${ }^{8}$ reported a series of patients with achalasia with normal IRP value; Sato et al ${ }^{9}$ also reported that $28.1 \%$ of patients showed IRP within the normal range. In addition, there are several studies reporting that a subset of patients with achalasia have normal IRP values. ${ }^{10-13}$

The aims of this study are to determine the clinical characteristics of a subset of patients with achalasia with normal IRP value, and to evaluate outcomes after peroral endoscopic myotomy (POEM) of this subgroup of patients.

\section{Materials and Methods}

\section{Study Participants}

For this study, patients who presented to our center between 2014 to 2018 with typical symptoms of achalasia were included. All patients were examined with upper endoscopy, esophagography, HRM, 24-hour ambulatory $\mathrm{pH}$ monitoring, and impedance planimetry. Structural obstructions such as malignancy and extrinsic compression were ruled out. Patients who were diagnosed with achalasia were treated with POEM. All patients provided informed consent before the procedure. Approval of the Institutional Review Board was received for this retrospective study (2018-07-050-001).

\section{Diagnostic Measurements}

\section{Eckardt symptom score}

The Eckardt score was used to assess patients' symptoms. This score is the sum of scores of dysphagia, chest pain, regurgitation (3, each meal; 2, daily; 1 , occasionally; 0 , absent), and weight loss ( $3,>$ $10 \mathrm{~kg} ; 2,5-10 \mathrm{~kg} ; 1,<5 \mathrm{~kg} ; 0$, no weight loss). ${ }^{14}$ The maximum score is 12 and the more severe the symptoms, the higher the score. Patients were requested to answer the questionnaires at the initial visit and 3 months, 6 months, and 1 year after POEM. When the Eckardt score was less than 3 points after treatment, the treatment was considered successful; a score greater than 3 points was considered a high score, indicating that additional treatment is needed. ${ }^{15}$

\section{Esophageal high-resolution manometry}

All patients were assessed with esophageal HRM at the time of diagnosis and 3 months, 6 months, and 1 year after POEM. Manometry used a HRM catheter (Sandhill Scientifics, Littleton, CO, USA) according to standard protocols. ${ }^{16}$ The upper normal limit of IRP was set as $20 \mathrm{mmHg}$, according to the recommendation of the manufacturer. Achalasia subtypes were determined according to the Chicago classification based on esophageal contraction and pressurization patterns: type I, failed peristalsis; type II, failed peristalsis with panesophageal pressurization in more than $20 \%$ of the swallows; and type III, absent peristalsis with spastic, premature contractions in more than $20 \%$ of the swallows. ${ }^{17}$

When HRM catheter could not pass through the LES, we inserted the catheter with an endoscopy guided method. In difficult cases, we checked catheter state with the fluoroscopy in the endoscopy unit.

\section{Timed barium esophagography}

Timed esophagogram was performed to assess esophageal emptying. Patients were asked to ingest a tolerable maximal amount of barium suspension ( $50 \mathrm{~mL}$ to $200 \mathrm{~mL}$ ) in a standing position. After ingestion of the barium suspension, real-time video was obtained for up to 5 minutes to determine esophageal morphology, peristalsis, and stasis at the esophagus. ${ }^{18}$ The distance from the top of the contrast column to the esophagogastric junction (EGJ) and the maximal esophageal diameter were measured. Height of the barium column at 5 minutes was used to determine esophageal emptying. ${ }^{19}$ 


\section{Esophagogastroduodenoscopy and endoscopic ultra- sound}

All patients underwent upper endoscopy at the time of diagnosis for exclusion of mechanical obstruction or other mucosal lesions. Esophageal muscle thickness was measured by endoscopic ultrasound. Follow-up endoscopy was performed at 3 months, 6 months, and 1 year after POEM for evaluation of EGJ tightness, mucosal lesions, and reflux esophagitis.

\section{Esophagogastric junction distensibility by impedance planimetry (EndoFLIP)}

For the measurement of EGJ distensibility, the impedance planimetry tool, endoscopic functional luminal imaging probe (EndoFLIP; Crospon Ltd, Galway, Ireland), was used. EndoFLIP uses impedance planimetry to measure cross-sectional areas (CSAs) in the esophagus. ${ }^{20}$ Distensibility index (DI) of the EGJ was determined by dividing the median minimal CSA by the median intrabag pressure at a given volume of distension during the 30 second recording period.

\section{Per-oral endoscopic myotomy}

All recruited patients with achalasia were treated with POEM. POEM was performed under general anesthesia based on the previously described technique by Inoue et $\mathrm{al}^{21} \mathrm{POEM}$ was performed by 2 experienced endoscopists. Briefly, mucosal incision and submucosal tunnel was made after submucosal injection at $9 \mathrm{~cm}$ to $10 \mathrm{~cm}$ above the EGJ with the endoscopic submucosal dissection technique. A selective myotomy of the circumferential muscle fibers was performed proximal portion. Then, full-thickness myotomy of both circumferential and longitudinal muscle fibers was performed from $5 \mathrm{~cm}$ proximal to $2 \mathrm{~cm}$ below the EGJ. The myotomy length was confirmed with the double-scope method ${ }^{22}$ and the incision site was closed with hemoclips.

\section{Statistical Methods}

Data are presented as mean $\pm \mathrm{SD}$. Statistical analysis was performed using IBM SPSS Statistics version 25 (IBM Corporation, Armonk, NY, USA). Clinical characteristics were compared using the chi-squared test for categorical data and the Student's $t$ test for continuous. Associations between parameters were evaluated with Spearman's correlation analysis. Differences were considered statistically significant when $P$ is below 0.05 .

\section{Results}

From November 2014 to April 2018, 155 patients underwent POEM. Patients with other esophageal motility disorders such as jackhammer esophagus, distal esophageal spasm, EGJ outflow obstruction, and patients with atypical patterns on HRM were excluded. Patients who had been previously treated with botulinum toxin injections, Heller-myotomy, pneumatic balloon dilatations, and POEM were also excluded. When all the results of studies were compatible with achalasia except normal IRP values and when other esophageal motility disorders were ruled out, these patients were diagnosed as achalasia with normal IRP and managed with POEM. Thus, 89 patients were included in the analysis. The patients were sub-classified into 2 groups according to IRP: 65 patients $(73 \%)$ showed impaired EGJ relaxation and 24 patients $(27 \%)$ showed normal EGJ relaxation.

\section{Clinical Characteristics and Initial Presentation}

Table 1 summarizes patients' clinical characteristics and diagnostic results before POEM. In the normal IRP value group (IRP $\leq 20 \mathrm{mmHg}$ ), patients were older and had a more tortuous esophagus $(P=0.001$ and 0.049 , respectively). This group also showed trends toward longer symptom duration and increased prevalence of achalasia type I, but without statistical significance. Sex, body mass index (BMI), mean esophageal diameters, and Eckardt scores were indifferent between the 2 groups.

DI and CSAs measured with EndoFlip were higher in the normal IRP value group. LES muscle thickness was indifferent between 2 groups.

\section{Therapeutic Outcomes of Peroral Endoscopic Myotomy}

In the normal IRP value group, Eckardt scores significantly decreased from $6.47 \pm 2.87$ to $1.41 \pm 1.06$ with a significant reduction in IRP. Similarly, in the impaired LES relaxation group, the Eckardt scores decreased from $6.48 \pm 2.28$ to $1.43 \pm 1.34$, and IRP also decreased significantly (Fig. 1). Eckardt scores were indifferent but DI was significantly different between groups before and after POEM (Tables 1 and 2).

\section{Correlation Between Integrated Relaxation Pressure and Other Factors}

We examined the IRP-related parameters through correlation analysis. Of the various parameters, age, symptom duration, and DI 
Table 1. Clinical Characteristics of Patients With Achalasia According to the Values of Integrated Relaxation Pressure

\begin{tabular}{|c|c|c|c|}
\hline Parameters & Normal IRP group $(\mathrm{n}=24)$ & Abnormal IRP group $(\mathrm{n}=65)$ & $P$-value \\
\hline Sex ratio (male:female) & $11: 13$ & $34: 31$ & 0.588 \\
\hline Age (yr) & $51.12 \pm 15.51$ & $39.80 \pm 13.82$ & $<0.001$ \\
\hline Body mass index $\left(\mathrm{kg} / \mathrm{m}^{2}\right)$ & $22.97 \pm 3.42$ & $22.28 \pm 3.36$ & 0.395 \\
\hline Duration of symptom (yr) & $6.31 \pm 6.94$ & $3.94 \pm 5.65$ & 0.102 \\
\hline Eckardt score & $6.47 \pm 2.87$ & $6.48 \pm 2.28$ & 0.991 \\
\hline Achalasia type (I:II:III) (n) & $11: 12: 1$ & $17: 37: 11$ & 0.109 \\
\hline Maximal esophageal diameter $(\mathrm{cm})$ & $3.95 \pm 1.89$ & $3.61 \pm 1.15$ & 0.322 \\
\hline Sigmoid type & $4(16.6)$ & $3(4.6)$ & 0.049 \\
\hline IRP (mmHg) & $11.5 \pm 6.46$ & $38.11 \pm 21.36$ & $<0.001$ \\
\hline Residual LES pressure (mmHg) & $16.75 \pm 13.44$ & $42.22 \pm 17.00$ & $<0.001$ \\
\hline Distensibility index $\left(\mathrm{mm}^{2} / \mathrm{mmHg}\right)$ & $2.75 \pm 3.15$ & $1.56 \pm 1.04$ & 0.025 \\
\hline Cross sectional area $\left(\mathrm{mm}^{2}\right)$ & $82.40 \pm 90.29$ & $49.20 \pm 26.28$ & 0.024 \\
\hline EGJ muscle thickness (mm) & $2.96 \pm 1.24$ & $2.64 \pm 1.10$ & 0.302 \\
\hline Inner circular muscle thickness (mm) & $1.72 \pm 0.95$ & $1.49 \pm 0.82$ & 0.309 \\
\hline Operation time ( $\mathrm{min})$ & $84.45 \pm 33.95$ & $75.01 \pm 31.71$ & 0.226 \\
\hline Myotomy length (cm) & $8.86 \pm 2.99$ & $9.42 \pm 1.98$ & 0.333 \\
\hline
\end{tabular}

IRP, integrated relaxation pressure; LES, lower esophageal sphincter; EGJ, esophagogastric junction.

Data are presented as mean $\pm \mathrm{SD}$ or number $(\%)$.
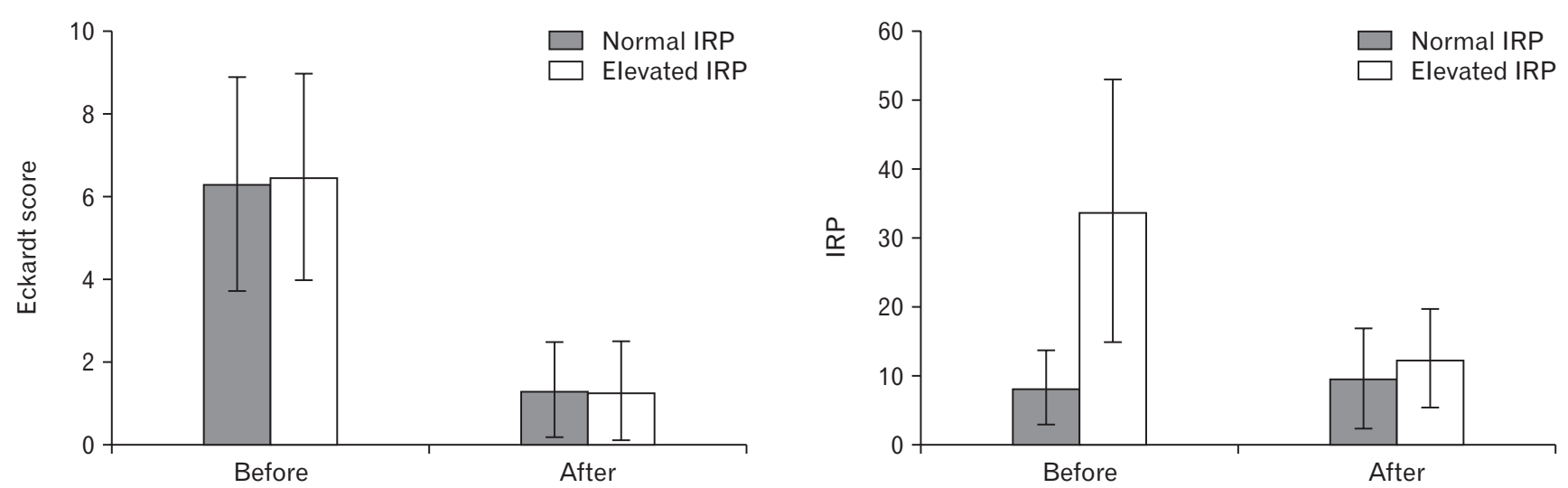

Figure 1. Therapeutic outcomes of peroral endoscopic myotomy (POEM) in 2 groups. The Eckardt score significantly decreased after POEM in both groups. Integrated relaxation pressure (IRP) decreased to the normal range after POEM in the elevated IRP group but was not different in the normal IRP group.

Table 2. Therapeutic Outcomes After Peroral Endoscopic Myotomy

\begin{tabular}{lccc}
\hline \multicolumn{1}{c}{ Parameters } & Normal IRP group $(\mathrm{n}=24)$ & Abnormal IRP group $(\mathrm{n}=65)$ & $P$-value \\
\hline Eckardt score & $1.36 \pm 1.18$ & $1.30 \pm 1.18$ & 0.835 \\
IRP $(\mathrm{mmHg})$ & $9.77 \pm 7.47$ & $12.41 \pm 7.07$ & 0.176 \\
Residual LES pressure $(\mathrm{mmHg})$ & $12.77 \pm 9.35$ & $14.70 \pm 9.42$ & 0.439 \\
Distensibility index $\left(\mathrm{mm}^{2} / \mathrm{mmHg}\right)$ & $13.89 \pm 7.71$ & $10.16 \pm 6.68$ & 0.027 \\
Cross sectional area $\left(\mathrm{mm}^{2}\right)$ & $271.34 \pm 118.33$ & $256.69 \pm 126.98$ & 0.645 \\
Clinically improved after $1 \mathrm{yr}$ & $23 / 24(95.8)$ & $63 / 65(96.9)$ & 0.991 \\
\hline
\end{tabular}

IRP, integrated relaxation pressure; LES, lower esophageal sphincter.

Data are presented as mean $\pm \mathrm{SD}$ or number $(\%)$. 

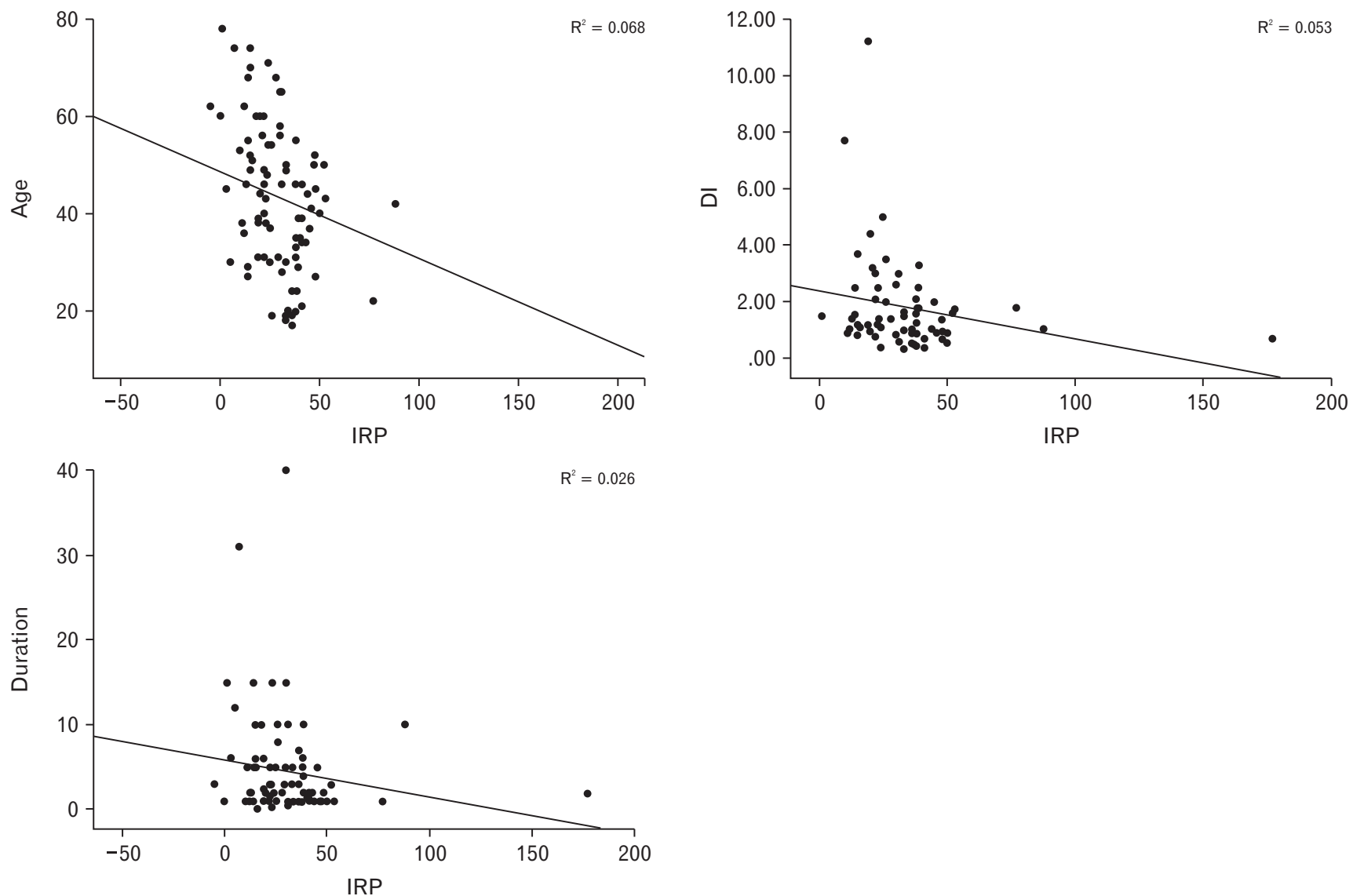

Figure 2. Correlation between integrated relaxation pressure (IRP) and other factors in patients with achalasia. Age, symptom duration, and distensibility index (DI) showed negative correlation with IRP.

showed negative correlations with IRP (Fig. 2). BMI, esophageal diameter, and Eckardt scores did not have significant correlation with IRP (Table 3). There were some correlations among parameters such as age and BMI, BMI and Eckardt score, and symptom duration and esophageal diameter.

\section{Discussion}

This study suggests that patients with clinical features of achalasia but with normal IRP in HRM were older and had a more tortuous esophagus and higher EGJ distensibility. These patients showed trends without statistical significance toward longer symptom duration and different composition of achalasia subtypes.

The IRP has been proven to be the best parameter to evaluate EGJ function and to predict abnormal transmission at this level, but there is limited data on the association between the IRP value and patients' characteristics. ${ }^{17}$ According to the Chicago classification version 3.0, patients with IRP in the normal range but with $100 \%$
Table 3. Correlations Between Integrated Relaxation Pressure and Other Parameters

\begin{tabular}{lcr}
\hline \multicolumn{1}{c}{ Parameters } & Correlation coefficient & $P$-value \\
\hline Age & $-0.37^{\mathrm{a}}$ & $<0.001$ \\
Body mass index & -0.18 & 0.098 \\
Symptom duration & $-0.22^{\mathrm{b}}$ & 0.040 \\
Esophageal diameter & -0.04 & 0.703 \\
Distensibility index & $-0.27^{\mathrm{b}}$ & 0.031 \\
Eckardt score & 0.06 & 0.625 \\
\hline${ }^{a} P<0.01,{ }^{\mathrm{b}} P<0.05$ & &
\end{tabular}

failed peristalsis are classified as having absent contractility. Absent contractility is usually accompanied by connective tissue disease, which is difficult to treat and has a poor prognosis. ${ }^{23}$ Its pathophysiology is not clearly identified, but might be related to extracellular matrix degradation, vascular disorders, and autoantibodies, which are different pathomechanisms from achalasia. ${ }^{24}$ The Chicago classification states that patients with failed peristalsis and borderline 
IRP could potentially have achalasia.

Patients with type I achalasia had severe fibrosis of the esophageal muscle layer compared with patients with type II achalasia, based on surgical biopsy. ${ }^{25}$ Interstitial cells of Cajal were preserved in type III achalasia, which showed mild atrophy and fibrosis, indicating that the etiology of the different achalasia types might be different. There was a negative correlation between IRP and age, showing severe fibrosis in patients with low IRP, and IRP could be normal due to low LES pressure with disease progression in patients with achalasia. ${ }^{9}$ When the more severe fibrosis occurs, the value of DI is usually lower. However, fibrosis itself of LES may decrease the residual LES pressure of achalasia and preserve CSA like this study. The residual LES pressure was lower in the normal IRP group and CSA of normal IRP group was larger than abnormal IRP group. DI of patient with larger CSA would be higher at first to some point of area and resultingly average value of DI of higher CSA group would be higher than that of small CSA group. This is in accordance with the current observations: our study also shows that patients with achalasia with normal IRP were older and had longer disease duration.

The physiology of the EGJ is complex and not fully understood. Its function depends on the interaction of the lower esophageal sphincter, the sphrenoesophageal ligament, sling fibers of the proximal stomach, and the crural diaphragm. ${ }^{26}$ IRP is dependent on the timing and pattern of distal esophageal contractility. IRP could be within the normal range without distal esophageal contraction and with low intra-esophageal pressure. ${ }^{27}$ The IRP cutoff could be different according to achalasia subtypes, that is, lower in type I than that of type III.

Patients with achalasia features but normal IRP value on HRM had impaired EGJ distensibility on EndoFLIP measurements. ${ }^{8}$ In the present study, EGJ distensibility measured by EndoFLIP was impaired in both groups. Patients with normal IRP value on HRM also showed impaired EGJ distensibility, with a slightly higher index than the impaired EGJ relaxation group. This might be explained by the presence of fibrosis of the LES muscle. In our study, the 2 groups had different distributions of achalasia subtypes. In the normal IRP value group, $46 \%$ had type I achalasia compared with $26 \%$ in the impaired LES relaxation group, this subtype differences also influence HRM results.

The use of impedance planimetry could be helpful for the diagnosis of achalasia with normal IRP value on HRM. The distensibility of the EGJ in patients with achalasia has been studied. ${ }^{2-4}$ Functional luminal imaging probe (FLIP) topography results could be discordant with HRM and may indicate undetected esophageal motility abnormalities. ${ }^{28}$ The presence and patterns of contractility such as repetitive antegrade contraction or repetitive retrograde contraction in the FLIP topography may be helpful for the detection of achalasia with normal IRP. ${ }^{29}$

The esophageal muscle is thickened in patients with esophageal motility disorder, and the thickness of the longitudinal and circular muscle layers increases in patients with achalasia. ${ }^{30}$ When the esophageal lumen is increased in patients with achalasia, the stretch effect may reduce muscle thickness, however, there was a report that muscle thickness in elderly patients was thicker than that of the younger aged. ${ }^{31}$ In this study, there was no difference of muscle thickness in endoscopic ultrasound findings between the 2 groups. It might be explained by a combined result from aging and stretch effect with long symptom duration. There was no difference in distal contractile integral between the 2 groups. Distal contractile integral is not important factor in achalasia and depends on the proportion of type III achalasia.

This study has several limitations. First, there is possibility to miss the true difference between the 2 groups due to small sample size. Second, this study included patients of a single race in a single center and might not reflect ethnic differences.

In conclusion, patients with achalasia with normal IRP value were older and had longer disease duration and higher DI than patients with impaired LES relaxation. Therapeutic outcomes were indifferent between the 2 patient groups. Therefore, patients with normal IRP also should be considered to be achalasia, and POEM is also effective in these patients.

\section{Financial support: None.}

\section{Conflicts of interest: None.}

Author contributions: Eunju Kim and In Kyung Yoo collected and analyzed data, and described manuscript and contributed equally to this work as first authors; Dong Keon Yon advised statistical analysis; Joo Young Cho and In Kyung Yoo recruited patients and performed POEM; and Sung Pyo Hong supervised the study and extremely revised the manuscript.

\section{References}

1. Mayberry JF. Epidemiology and demographics of achalasia. Gastrointest Endosc Clin N Am 2001;11:235-248.

2. Richter JE. Achalasia - an update. J Neurogastroenterol Motil 2010;16:232-242.

3. Boeckxstaens GE, Zaninotto G, Richter JE. Achalasia. Lancet 2014;383:83-93. 
4. Vaezi MF, Pandolfino JE, Vela MF. ACG clinical guideline: diagnosis and management of achalasia. Am J Gastroenterol 2013;108:1238-1249.

5. Ghoshal UC, Daschakraborty SB, Singh R. Pathogenesis of achalasia cardia. World J Gastroenterol 2012;18:3050-3057.

6. Park W, Vaezi MF. Etiology and pathogenesis of achalasia: the current understanding. Am J Gastroenterol 2005;100:1404-1414.

7. Kahrilas PJ, Bredenoord AJ, Fox M, et al. The Chicago classification of esophageal motility disorders, v3.0. Neurogastroenterol Motil 2015;27:160-174.

8. Ponds FA, Bredenoord AJ, Kessing BF, Smout AJ. Esophagogastric junction distensibility identifies achalasia subgroup with manometrically normal esophagogastric junction relaxation. Neurogastroenterol Motil 2017;29:e12908.

9. Sato H, Takahashi K, Mizuno KI, Hashimoto S, Yokoyama J, Terai S. A clinical study of peroral endoscopic myotomy reveals that impaired lower esophageal sphincter relaxation in achalasia is not only defined by highresolution manometry. PLoS One 2018;13:e0195423.

10. Mearin F, Malagelada JR. Complete lower esophageal sphincter relaxation observed in some achalasia patients is functionally inadequate. Am J Physiol Gastrointest Liver Physiol 2000;278:G376-G383.

11. Kim JH, Rhee PL, Lee SS, et al. Is aperistalsis with complete lower esophageal sphincter relaxation an early stage of classic achalasia? J Gastroenterol Hepatol 2007;22:536-541.

12. Hirano I, Tatum RP, Shi G, Sang Q, Joehl RJ, Kahrilas PJ. Manometric heterogeneity in patients with idiopathic achalasia. Gastroenterol 2001;120:789-798.

13. Katz PO, Richter JE, Cowan R, Castell DO. Apparent complete lower esophageal sphincter relaxation in achalasia. Gastroenterology 1986;90:978-983.

14. Eckardt AJ, Eckardt VF. Current clinical approach to achalasia. World J Gastroenterol 2009;15:3969-3975.

15. Krill JT, Naik RD, Vaezi MF. Clinical management of achalasia: current state of the art. Clin Exp Gastroenterol 2016;9:71-82.

16. do Carmo GC, Jafari J, Sifrim D, de Oliveira RB. Normal esophageal pressure topography metrics for data derived from the Sandhill Unisensor high resolution manometry assembly in supine and sitting positions. Neurogastroenterol Motil 2015;27:285-292.

17. Kahrilas PJ, Bredenoord AJ, Fox M, et al. The Chicago classification of esophageal motility disorders, v3.0. Neurogastroenterol Motil 2015;27:160-174.

18. de Oliveira JM, Birgisson S, Doinoff C, et al. Timed barium swallow: a simple technique for evaluating esophageal emptying in patients with achalasia. AJR Am J Roentgenol 1997;169:473-479.

19. Vaezi MF, Baker ME, Achkar E, Richter JE. Timed barium oesophagram: better predictor of long term success after pneumatic dilation in achalasia than symptom assessment. Gut 2002;50:765-770.

20. Kwiatek MA, Pandolfino JE, Hirano I, Kahrilas PJ. Esophagogastric junction distensibility assessed with an endoscopic functional luminal imaging probe (EndoFLIP). Gastrointest Endosc 2010;72:272-278.

21. Inoue $\mathrm{H}$, Sato H, Ikeda H, et al. Per-oral endoscopic myotomy: a series of 500 patients. J Am Coll Surg 2015;221:256-264.

22. Hong HJ, Song GW, Ko WJ, et al. Double-scope peroral endoscopic myotomy (POEM) for esophageal achalasia: the first trial of a new double-scope POEM. Clin Endosc 2016;49:383-386.

23. Nica AE, Alexa LM, Ionescu AO, Andronic O, Păduraru DN. Esophageal disorders in mixed connective tissue diseases. J Med Life 2016;9:141-143.

24. Reichlin M. Problems in differentiating SLE and mixed connectivetissue disease. N Engl J Med 1976;295:1194-1195.

25. Nakajima N, Sato H, Takahashi K, et al. Muscle layer histopathology and manometry pattern of primary esophageal motility disorders including achalasia. Neurogastroenterol Motil 2017;29:e12968.

26. Mittal RK, Balaban DH. The esophagogastric junction. N Engl J Med 1997;336:924-932.

27. Lin Z, Kahrilas PJ, Roman S, Boris L, Carlson D, Pandolfino JE. Refining the criterion for an abnormal integrated relaxation pressure in esophageal pressure topography based on the pattern of esophageal contractility using a classification and regression tree model. Neurogastroenterol Motil 2012;24:e356-e363.

28. Carlson DA, Kahrilas PJ, Lin Z, et al. Evaluation of esophageal motility utilizing the functional lumen imaging probe. Am J Gastroenterol 2016;111:1726-1735.

29. Carlson DA, Lin Z, Kahrilas PJ, et al. The functional lumen imaging probe detects esophageal contractility not observed with manometry in patients with achalasia. Gastroenterology 2015;149:1742-1751.

30. Puckett JL, Bhalla V, Liu J, Kassab G, Mittal RK. Oesophageal wall stress and muscle hypertrophy in high amplitude oesophageal contractions. Neurogastroenterol Motil 2005;17:791-799.

31. Dogan I, Puckett JL, Padda BS, Mittal RK. Prevalence of increased esophageal muscle thickness in patients with esophageal symptoms. Am J Gastroenterol 2007;102:137-145. 